\title{
利用桑想丝素蛋白制葡萄糖氧化酶 传感器的研究*
}

\author{
方 跃正中 那家視 于同隐** \\ (复旦大学化学系、材料科学系, 上海 200433)
}

关链词程丝素蛋白、蛋白质变性、酶固定化、传感器

生物活性酶的固定化是几乎所有类型的生物传感器制备过程中必经的步骤。固定化酶的 质量, 除与生物活性物质本身有关外, 固定化技术是一项重要的因素. 本文以葡萄糖缽化

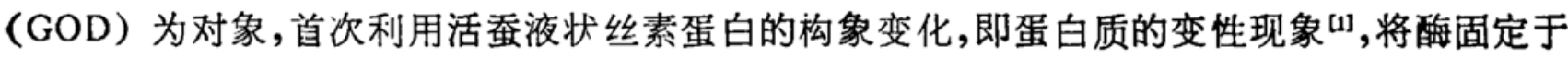
秦丝素蛋白中. 用这种新的酶国定化方法所制成的葡萄糖氧化酶传感器, 不仅具有制作方便, 酶活性降低少, 电极稳定性高, 响应速惨快, 寿命长等特点, 而且呈现优越的耐热能力. 同时, 对于同一批制备的酶一一丝素蛋白样品, 所制电极的性能有着相当好的重现性, 因而具有商品 化应用前景.

\section{一、实 验}

1. 原料所用的试剂均为分析纯，末经特殊处理．实验用水为高纯去离子水。葡萄糖 篻化酶 (GOD) 的活性为 $10 \mathrm{kU} / 46 \mathrm{mg}$. 家亘品种为浙 $12 \times$ 苏 1 , 由浙江农业大学提供.

2.GOD 在䖽液状丝秦皿白上的固定 将昍丝前 $12-24 \mathrm{~h}$ 的熟羔解剖, 取中部丝腺体, f 细剥离腺体膜组织, 得到的胶状丝素䖤白经去离子水充分洗涤,除去丝胶部分, 并使之在水 中缓慢溶解, 制成约 $2 \%$ 的水溶液, $5 \mathrm{mg}$ GOD 溶于 $5 \mathrm{ml}$ 水中, 与 $50 \mathrm{ml}$ 的丝素蛋白水溶液充 分混合, 在聚乙烯上用以下两种方法固定化: (1) 室温下, 真空 $48 \mathrm{~h}$ 成膜, 膜厚约 $20 \mu \mathrm{m}$, 将膜 在甲醇中浸泡 $30 \mathrm{~min}$, 使丝素蛋白变性, 取出迅速使甲醇挥发, 制得 GOD-丝素膜. (2) 室温 下,真空内放置 $6 \mathrm{~h}$, 使丝索蛋白-GOD 溶液浓缩成 $50 \%$ 左右的胶状液, 放入 $-20{ }^{\circ} \mathrm{C}$ 以下的环 境中, 剩余的水份迅速结冰, $24 \mathrm{~h}$ 后取出并室温干焻。得到粉末状样品, 称为 GOD-丝秦粉 末.

3. 丝素蛋白的构象测定 用 Spex 1403 激光 Raman 仪, 氞离子激光器 (Spectra Physics Model Sp-164) 对丝素蛋白样品进行 Raman 光谱的测定, 记录扫描范围: 800一 $1800 \mathrm{~cm}^{-1}$.

4. 传感器的制备及性能测试 GOD-丝素膜或粉末在 $4^{\circ} \mathrm{C}, 0.1 \mathrm{~mol} / \mathrm{L} \mathrm{pH}=7.0$ 的磷酸 盐缓冲液中浸泡 $24 \mathrm{~h}$, 除尽未固定的 GOD, 取出晾干, 称 $2 \mathrm{mg}$ 左右的酶膜或粉末,外层用 81

本女1991年1月22 日收到. 1991 年 6 月 13 日收到改改。

* 国家自然科学基金资助项目.

**通讯联系人.

第 4 期

科学 通 报

327 
（240）目单丝尼龙网按“三明治”式夹膜法固定在氧电极上,制成㑘萄糖传感器。

移取 $10.0 \mathrm{ml}$ 缓冲液于 $25 \mathrm{ml}$ 干燥烧杯中,加 $0.05 \mathrm{ml} 10^{-2} \mathrm{~mol} / \mathrm{L}$ EDTA 溶液, 插入传感器, 恒速擋拌, 待读数稳定后,加一定体积的菏苟糖标准液,用测氧仪(中国科学院上海冶金研究所) 配合 3086 型 X-Y 函数记录仪 (日本横河公司, 重庆组装) 记录各浓度下的信号值 $E(\mathrm{mV})$, 以 $\Delta E$ 对浓度 $c$ 作图,绘制标准工作曲线.

\section{二、结果和讨论}

1. GOD 在洨状丝羕蛋白上的固定化机理通常认为, 成熟桑虫体内中部夜状丝絭蛋

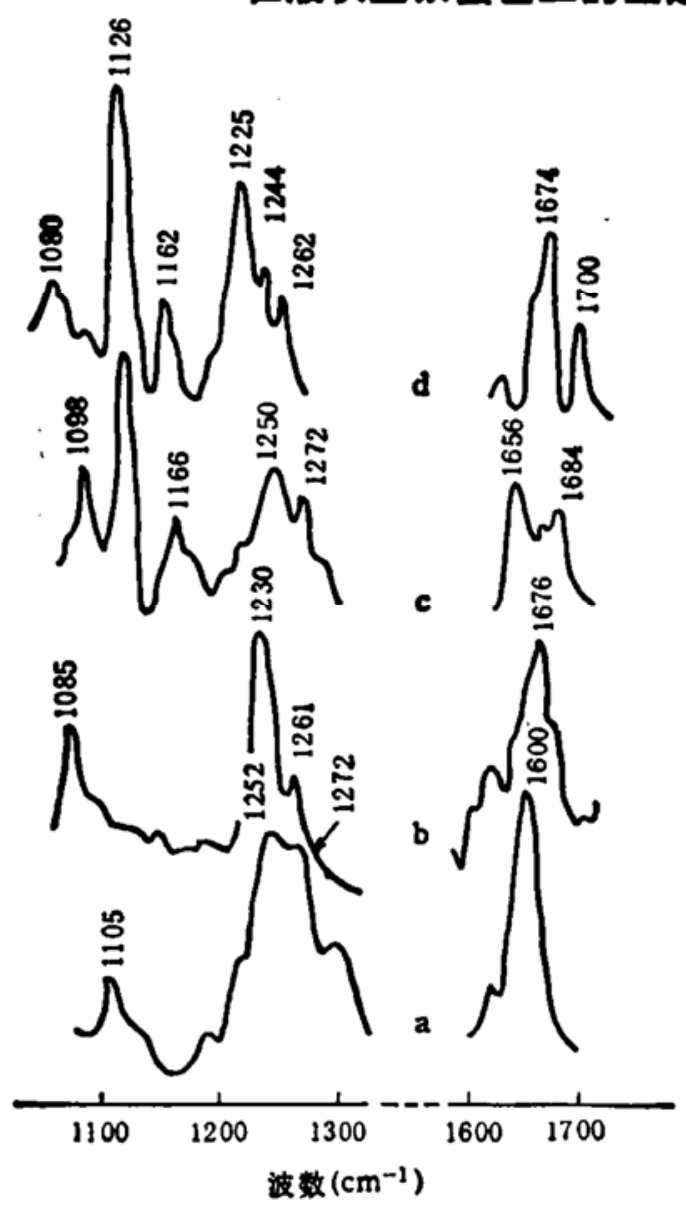

图 1 不同条件下,丝索蛋白的 Raman 光谱

a. 旅状丝素虽白; b. 冰练后的丝素蛋白; c. GOD-丝素膜, 未用甲酸处理; d. GOD丝絭䠑甲醇处理 $30 \mathrm{~min}$

白的构象以可溶性的 $\alpha$-螺旋和无规线团为主 ${ }^{[1]}$. 当 其以一定的浓度, 在确定的条件下成膜时, 将会生成 不同的结构形态 ${ }^{[2]}$. 通过对宴液状丝素蛋白纤维化 机理的研究, 人们发现不仅拉伸或剪切作用能够导 致丝素蛋白的构象向 $\beta$-折叠转变, 并且在如加热、甲 醇漫泡等过程作用后, 具有 $\alpha$-螺旋和无定形态的丝 素膜也将产生不溶性的 $\rho^{3}$-折叠结构 ${ }^{[3]}$. 实验中发现, 当液状丝素蛋白被冷冻时, 内含的水在由液态向固 态转化过程中, 将对丝素蛋白产生一个足够的剪切 挤压力,也能导致丝蛋白的变性. 我们用 Raman 光 谱测定了在不同条件下, 丝素蛋白的构象变化（图 1). 冷冻或甲醇处理后, 在 Amide III 区域, 标志 着 $\alpha$-螺旋和无规线团的 $1100,1252,1270 \mathrm{~cm}^{-1}$ 特 征谱带 ${ }^{[4-6]}$ 强度降低甚至消失, 而在 $1080,1230 \mathrm{~cm}^{-1}$ 附近出现 $\beta$-折叠的特征谱带. 同时, Amide I 区 域原有的 $1660 \mathrm{~cm}^{-1}$ 附近谱带移至 $\beta$-折叠的特征 位 置 $1676 \mathrm{~cm}^{-1}$ 处. 这表明, 通过冷冻或甲醇处理过 程,丝素蛋白的构象已从 $\alpha$-螺旋和无规线团变成 $\beta-$ 折叠. 由于 $\beta$-折叠的形成, 其分子间氢键所具备的 特定空间能将生物酶固定于其中. 这种固定化技术 不涉及或涉及很少的化学试剂, 因而所制成的酶传 感器在各项性能上具有独特的效果. 实验结果表 明, GOD-丝素膜在甲醇中浸泡 $30 \mathrm{~min}$ 效果最佳, 酶活力达 $12.6 \mathrm{U} / \mathrm{mg}$; 而在 GOD-丝素粉末中, 酶活力为 $20.1 \mathrm{U} / \mathrm{mg}$.

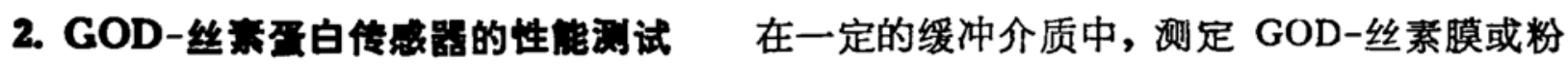
末传感器对同一荡萄螗标准液在各 pH 值下的响应. 图 2 表明, 在酸性工作条件下, GOD丝紊粉末传感器的相对活性比 GOD-丝素膜传感器稍高, 但两者随 $\mathrm{pH}$ 值变化的趋势一 致. 碱性条件下, GOD-丝素枌末传感器的活性几乎不变, 而 GOD-丝索膜传感器的相对活 性则有明显的下降. 考虑到传感器的响应时间随工作介质碱性的增大略有增长, 因此选用 $\mathrm{pH}-7.0$ 作为传感器的工作 $\mathrm{pH}$ 值.

在 $25 \%, \mathrm{pH}-7.0$ 的磷酸盐缓冲液中, 通过连续加样法测得 GOD-丝素膜或粉末传感器 
的标准工作曲线 (图 3), 其各项性能指标列于表 1.

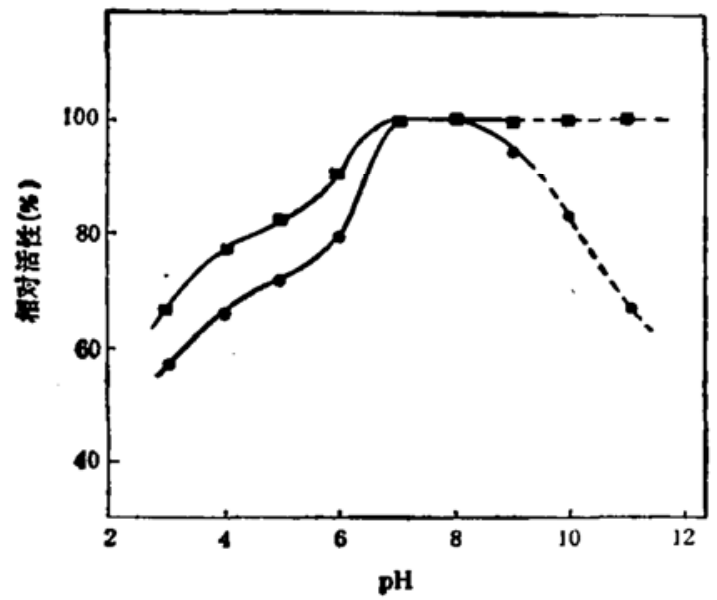

图 2 工作介质的 $\mathrm{pH}$ 值对传感器相对活性的影响

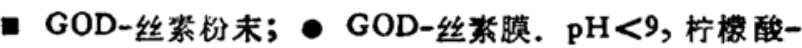
$\mathrm{K}_{2} \mathrm{HPO}_{4}$ 缓冲液; $\mathrm{pH}>9, \mathrm{~K}_{2} \mathrm{HPO}_{4}-\mathrm{NaOH}$ 缓冲液

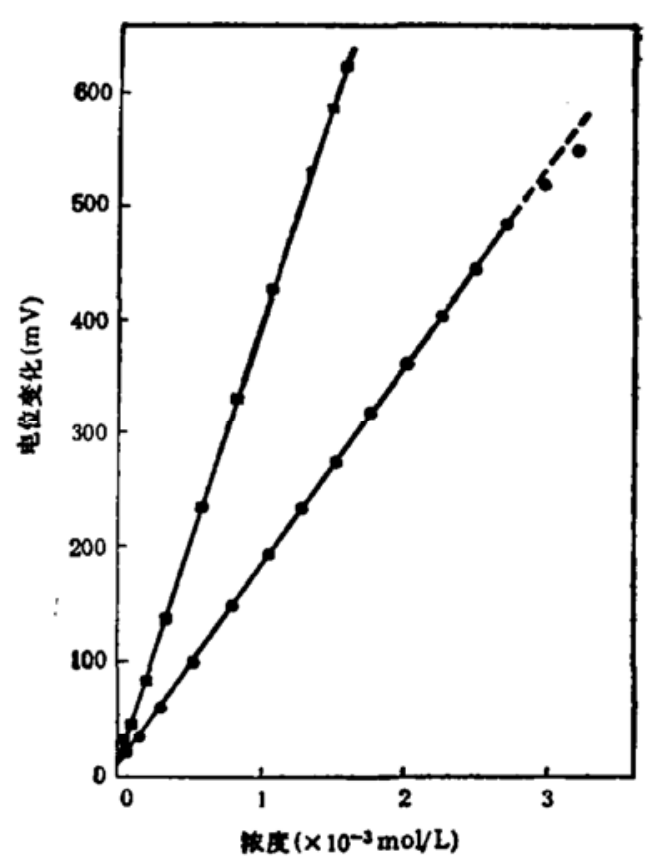

图 3 GOD-丝素蛋白传感器的标准工作曲线 - GOD-丝索粉末; - GOD-丝䒬瞙

表 1 GOD-丝素蛋白传感器的响应特性

\begin{tabular}{l|c|c}
\hline \multicolumn{1}{c|}{ 传 感器 } & GOD-丝索膜 & GOD-丝系粉末 \\
\hline 回归方程 & $\Delta E(\mathrm{mV})=6.27+1.78 \times 10^{3} C\left(\mathrm{~mol} \cdot \mathrm{L}^{-1}\right)$ & $\Delta E(\mathrm{mV})=3.63+3.95 \times 10^{\prime} C\left(\mathrm{~mol} \cdot \mathrm{L}^{-1}\right)$ \\
\hline 相关系数 & 0.9999 & 0.9999 \\
\hline 线珄范围 $\left(\mathrm{mol} \cdot \mathrm{L}^{-1}\right)$ & $1.5 \times 10^{-4}-2.5 \times 10^{-3}$ & $6.0 \times 10^{-3}-1.6 \times 10^{-3}$ \\
\hline 检测限 $\left(\mathrm{mol} \cdot \mathrm{L}^{-1}\right)$ & $5.0 \times 10^{-9}$ & $4.0 \times 10^{-3}$ \\
\hline 响应时间 $(\mathrm{min})$ & $1-1.5$ & $1-2.5$ \\
\hline
\end{tabular}

从图 3 和表 1 可以发现, 尽管用两种固定化方法所制得的传感器的工作性能均令人满意, 但 GOD-丝素粉末中的 GOD 活性仍要比 GOD-丝素膜高很多, 且它的检测限也较低, 这是 由于在 GOD-丝素粉末的固定化过程中, 未引人任何其它的化学物质, GOD 几乎保持原应 有的活性.

将 GOD-丝素膜或粉末传感器放人 $\mathrm{pH}-7.0$ 的磷酸盐缓冲液内, 在选定的温度下浸泡 $30 \mathrm{~min}$, 然后 $25^{\circ} \mathrm{C}$ 时测定其相对活性，结果列于表 2。同时实验表明，同量游离的 GOD 在 $50^{\circ} \mathrm{C}$ 漫泡后,其相对活性约下降 $70 \%, 60^{\circ} \mathrm{C}$ 处理后, 酶活性基本丧失. 因此, 当 GOD 被固 定在虽丝索蛋白中后, 耐热性能大大提高. $80^{\circ} \mathrm{C}$ 左右, GOD 在丝素蛋白中完全失活, 此温度 可能是 GOD-丝索蛋白的极限使用温度.

表 2 GOD-丝素蛋白传感器的徱热性能

\begin{tabular}{|c|c|c|c|c|c|c|}
\hline \multicolumn{2}{|c|}{ 渴度 $\pm^{1}\left({ }^{\circ} \mathrm{C}\right)$} & 25 & 40 & 60 & 70 & 80 \\
\hline \multirow{2}{*}{$\begin{array}{c}\text { 相对活性 } \\
\text { (\%) }\end{array}$} & GOD-丝素膜 & 100.0 & 101.9 & 100.0 & 98.5 & 0 \\
\hline & GOD-丝索粉末 & 100.0 & 100.1 & 98.5 & 60.0 & 9.8 \\
\hline
\end{tabular}


两种固定化方法制成的传感器对水溶液中淢苟糖合成样品的回收率试验 结果列于表 3 表4. GOD-丝素膜和GOD-丝素粉末传感器的平均回收率以及平均相对偏差分别为 $100.2 \%$, $2.0 \% ; 99.8 \%, 1.7 \%$.

表 3 GOD-丝素膜对菊萄糖溶液的回收率 $\left(25 \pm 0.1^{\circ} \mathrm{C}\right.$, 磷酸盐缓冲液, $\mathrm{pH}=7.0$ 与表 4 同)

\begin{tabular}{c|c|c|c|c|c|c|c|c|c|c|c|c|c|c|}
\hline $\begin{array}{c}\text { 浓度 } \\
\left(\mathrm{mol} \cdot \mathrm{L}^{-1}\right)\end{array}$ & $\begin{array}{c}4.43 \times \\
10^{-4}\end{array}$ & $\begin{array}{c}4.98 \times \\
10^{-4}\end{array}$ & $\begin{array}{c}6.14 \times \\
10^{-4}\end{array}$ & $\begin{array}{c}7.35 \times \\
10^{-4}\end{array}$ & $\begin{array}{c}8.47 \times \\
10^{-4}\end{array}$ & $\begin{array}{c}9.90 \times \\
10^{-4}\end{array}$ & $\begin{array}{c}1.24 \times \\
10^{-3}\end{array}$ & $\begin{array}{c}1.49 \times \\
10^{-3}\end{array}$ & $\begin{array}{c}1.98 \times \\
10^{-3}\end{array}$ & $\begin{array}{c}2.48 \times \\
10^{-4}\end{array}$ \\
\hline 回收束(\%) & 99.7 & 96.0 & 99.0 & 100.4 & 100.0 & 96.9 & 98.9 & 104.9 & 103.6 & 99.9 \\
\hline
\end{tabular}

表 4 GOD-丝素粉末对蒱萄楉溶液的回收率

\begin{tabular}{c|c|c|c|c|c|c|c|c|c|c|c|c|}
\hline $\begin{array}{c}\text { 浓度 } \\
\left(\mathrm{mol} \cdot \mathrm{L}^{-1}\right)\end{array}$ & $\begin{array}{c}6.96 \times \\
10^{-9}\end{array}$ & $\begin{array}{c}9.82 \times \\
10^{-3}\end{array}$ & $\begin{array}{c}1.97 \times \\
10^{-4}\end{array}$ & $\begin{array}{c}2.48 \times \\
10^{-4}\end{array}$ & $\begin{array}{c}3.45 \times \\
10^{-4}\end{array}$ & $\begin{array}{c}4.95 \times \\
10^{-4}\end{array}$ & $\begin{array}{c}6.43 \times \\
10^{-4}\end{array}$ & $\begin{array}{c}7.85 \times \\
10^{-4}\end{array}$ & $\begin{array}{c}9.85 \times \\
10^{-4}\end{array}$ & $\begin{array}{c}1.23 \times \\
10^{-3}\end{array}$ & $\begin{array}{c}1.47 \times \\
10^{-3}\end{array}$ \\
\hline 回收束(\%) & 104.4 & 97.1 & 101.8 & 100.6 & 101.3 & 98.3 & 100.0 & 98.7 & 100.3 & 97.2 & 97.8 \\
\hline
\end{tabular}

GOD-丝素膜或粉末传感器置于 $\mathrm{pH}-7.0$ 的磷酸盐缓冲液中, 在 $4^{\circ} \mathrm{C}$ 的环境下储存, 每 天测工作曲线,一周后, 隔 6 天测定一次, 至今均达 3 个月以上, 传感器的各项指标基本保持不 变, 极限寿命仍在继续考查。此外, 将 GOD-丝素膜或粉末置于室温中, 2 个月后, GOD 的 活性没有降低. 并且对于同一批制备的 GOD-丝素膜或粉末,若均称取 $2 \mathrm{mg}$ 制成若千个传感 器,其响应特性可达到相当好的重现性,因而具有实现商品化的可能性.

这种新型的酶固定化方法, 不仅适用于 GOD 的固定, 而且还能应用于其它所有不破坏 蚕丝素蛋白的一级或二级分子结构的可溶性酶.

[1] Li Guangxian and Yu Tongyin, Chinese Science Bulletin, 35(1990). 857.

[2]于同隐、郡正中,滈分于通报,3(1990),154.

[ 3] Magoshi, J. et al., J. Polym. Sci.. Polym. Phys. Ed, 19(1981), 185.

[4] Tu, A. T., Ramen Spectroscopy in Biology: Princtples and Applicatiom. John Wiley, New York. 1982.

[5] Frushour, B. G. and Koenig, J. L., Biopolymers, 13(1974), 445.

[6] Itoh, K. and Oya, M., Polyen. J, 18(1986), 837. 